\title{
A characterization of parallelepipeds related to weak derivatives
}

\author{
Heinz Weisshaupt \\ University of Duesseldorf, Mathematical Institute \\ Universitaetsstrasse 1, D-40225 Duesseldorf, Germany \\ email: Heinz.Weisshaupt@uni-duesseldorf.de
}

July 2, 2003

\begin{abstract}
We characterize in this paper parallelepipeds in $\mathbb{R}^{m}$ within the family of all convex bodies by a property of special measures on its boundary. We show that these measures are related to weak derivatives (in the sense of [5] and [8]) of convex-valued functions. The results can be applied (see [9]) to derive a generalization of a theorem of Lehmann (see [4]) on the comparison of uniform location experiments.

Keywords: parallelepipeds, convex bodies, characterization, weak derivatives,
\end{abstract} convex-valued functions

AMS Subject Classification: 52A20, 52A38, 28A15, 26E25

\section{Introduction}

The investigation of special convex bodies and especially their characterization is a main topic in classical convex geometry (see [2] chapter 1.11). There exist several characterizations of parallelepipeds, simplexes and ellipsoids within the family of convex bodies (see [3], [1], [7] and [2]). In section 2 of this paper we give a new characterization of parallelepipeds by a property of certain measures on the boundary of convex sets. In section 3 we define measure valued weak derivatives of convex valued mappings. We show that the measures used to characterize parallelepipeds in section 2 are related to the derivatives of convex valued mappings given by shifts of the parallelepiped (convex body) in the directions of its 1-dimensional edges. We note that the results of section 3 can be used to prove a theorem on the comparison of uniform location experiments [9] which generalizes Theorem 3.1 of [4].

We denote by $\mathbb{N}=\{1,2, \ldots\}$ the set of positive integers. By $\mathbb{S}^{m-1}$ we denote the unit sphere in $\mathbb{R}^{m}$, by $\langle.,$.$\rangle we denote the euclidean scalar product on \mathbb{R}^{m}$ 
and by $\|.\|_{2}$ we denote the norm associated with $\langle.,$.$\rangle . By \bar{V}$ we denote the closure of a set $V$, by $\mathbf{1}_{B}$ we denote the indicator function of a set $B$ and by $\delta_{x}$ we denote the Dirac measure at a point $x$. Given a countable set $M$ we denote by $\delta_{M}$ the counting measure on $M$, i.e. $\delta_{M}(A)=\sum_{m \in M} \delta_{m}(A)$. Given two measures $\mu$ and $\nu$ on $\mathbb{R}^{m}$ we denote by $\mu * \nu$ the convolution of $\mu$ and $\nu$. If $\nu$ is absolutely continuous with respect to $\mu$ we write $\nu<<\mu$ and we denote by $\frac{d \nu}{d \mu}$ the Radon-Nykodim derivative of $\nu$ with respect to $\mu$. By $\lambda$ we denote Lebesgue measure on $\mathbb{R}^{m}$. Given a measure $\mu$ and a function $f$ we denote by $f \cdot \mu$ the measure defined by $[f \cdot \mu](A):=\int_{A} f d \mu$. We denote by af $f(C)$ the affine Hull of a set $C$. We say that a convex set $C$ is parallel to a convex set $D$ and write $C \| D$ if af $f(C) \subseteq$ af $f(D)+v$ or af $f(D) \subseteq$ af $f(C)+v$ for some $v \in \mathbb{R}^{m}$. Given a measure $\mu$ and a $\mu$-measurable set $Y$ we let $\left.\mu\right|_{Y}(A):=\mu(A \cap Y)$ denote the restriction of $\mu$ to the $\mu$-measurable subsets of $Y$.

\section{The characterization Theorem}

We state and prove in this section Theorem 1 which says that a convex body $C$ (a convex compact subset of $\mathbb{R}^{m}$ with nonempty interior) is a parallelepiped if and only if certain measures on the boundary $\partial C$ of $C$ possess a certain property.

Definition 1 Given a convex body $C \subset \mathbb{R}^{m}$ and $x \in \partial C$ we denote by $\nabla_{x} C$ the set of all hyper planes of support to $C$ at $x$. We denote by $\eta: \partial C \rightarrow \mathbb{S}^{m}$ an arbitrary mapping which maps the point $x \in \partial C$ to a vector $\eta(x)$ which is an outward unit normal vector to $C$ in $x$, i.e., $\langle\eta(x), y-x\rangle \leq 0$ and $\|\eta(x)\|_{2}=1$. Given a convex body $C$ we denote by ozC the surface area measure on $\partial C$.

Remark 1 By an application of Theorem 1.17 of [6] (which is a special case of Rademachers Theorem (Theorem 1.18 of [6]) there exists a set $D$ with oəC $(D)=$ 0 such that $\eta($.$) is uniquely defined on \partial C \backslash D$.

Definition 2 Given a vector $w \in \mathbb{R}^{m}$ and a convex body $C \subset \mathbb{R}^{m}$ we denote by $\varsigma_{w}^{C}$ the unique measure for which $\frac{d \varsigma_{w}^{C}}{d o \partial C}=\langle\eta(), w.\rangle \cdot o_{\partial C}$. By Remark 1 this definition is independent of the special choice of the function $\eta$ in Definition 1.

Theorem 1 A convex body $C \subset \mathbb{R}^{m}$ is a parallelepiped with one dimensional edges parallel $\left\{w_{1}, \ldots, w_{m}\right\}$ if and only if there exists a linearly independent set $\left\{w_{1}, \ldots, w_{m}\right\}$ of vectors $w_{j} \in \mathbb{R}^{m}$ such that for any $j \in\{1, \ldots, m\}$ there exists a countable set $R \subset \mathbb{R}$ and an $r_{0} \in R$ such that

$$
\left[\varsigma_{w_{j}}^{C} * \delta_{\left\{r \cdot w_{j} \mid r \in R\right\}}\right](B)=0
$$

for any Borel measurable set $B \subseteq \partial\left(C+r_{0} \cdot w_{j}\right)$, i.e. if and only if $\varsigma_{w_{j}}^{C} * \delta_{\left\{r \cdot w_{j} \mid r \in R\right\}}$ vanishes on the Borel measurable subsets of $\partial\left(C+r_{0} \cdot w_{j}\right)$. 
Proof: If $C$ is a parallelepiped we just have to choose $w_{i}$ such that $\left\|w_{i}\right\|$ equals the length of the one dimensional edge of $C$ which is parallel to $w_{i}$, $R=\{-1,0,1\}$ and $r_{0}=0$ independent of $j$. Then

$$
\left[\varsigma_{w_{j}}^{C} * \delta_{\left\{r \cdot w_{j} \mid r \in R\right\}}\right](B)=0 \text { for all Borel measurable sets } B \subseteq \partial\left(C+r_{0} \cdot w_{j}\right)
$$

which proves the only if part. The only if part is the consequence of the lemmas 1, 2 and 3 which we state and prove below.

Proposition 1 Given a convex body $C$, a vector $w \neq 0$, a real number $\beta_{1}$ and a point $x \in \partial C+\beta_{1} w$. If for arbitrary hyper planes $H$

$$
H \| w \Rightarrow H \notin \nabla_{x}\left[C+\beta_{1} w\right]
$$

then there exists one and only one $\beta_{2} \neq \beta_{1}$ such that $x \in \partial C+\beta_{2} w$.

Proof. We show that $x+\mathbb{R} w \cap \operatorname{int}\left(C+\beta_{1} w\right) \neq \emptyset$. Otherwise there would exist a hyperplane $H$ which separates $x+\mathbb{R} w$ and $C+\beta_{1} w$. This hyperplane would fulfill $H \in \nabla_{x}\left[C+\beta_{1} w\right]$ and $H \| w$ which contradicts our hypothesis. Thus there exists $y \in(x+\mathbb{R} w) \cap \operatorname{int}\left(C+\beta_{1} w\right)$. By compactness and convexity of $C$ we get that $(x+\mathbb{R} w) \cap\left(\partial C+\beta_{1} w\right)=(y+\mathbb{R} w) \cap\left(\partial C+\beta_{1} w\right)$ consists of exactly two points. One of them is $x$ the other one is $x+\gamma w$ for some $\gamma \in \mathbb{R}$. Thus for $\beta_{2} \neq \beta_{1}$ we have $x \in C+\beta_{2} w$ if and only if $\beta_{2}=\beta_{1}-\gamma$.

Proposition 2 Given a vector $w \neq 0$. Suppose that the intersection of a convex body $C$ and its translate $C+\alpha w$ (with $\alpha \neq 0$ ) is again a convex body, i.e., $\operatorname{int}(C \cap(C+\alpha w)) \neq \emptyset$. Then

$$
o_{\partial C}\left(\left\{x \mid x \in \partial C \cap(\partial C+\alpha w) \text { s.t. }\left[H \| w \Rightarrow H \notin \nabla_{x} C\right]\right\}\right)=0
$$

and

$$
o_{\partial C+\alpha w}\left(\left\{x \mid x \in \partial C \cap(\partial C+\alpha w) \text { s.t. }\left[H \| w \Rightarrow H \notin \nabla_{x} C\right]\right\}\right)=0
$$

Proof. We prove only (1), since the proof of (2) is completely analogous. Let

$$
X=\left\{x \mid x \in \partial C \cap(\partial C+\alpha w) \text { s.t. }\left[H \| w \Rightarrow H \notin \nabla_{x} C\right]\right\} .
$$

We have to show that $o_{\partial C}(X)=0$. We show first that $x \in X$ implies that

$$
(x+\mathbb{R} w) \cap X=x .
$$

We prove (3) indirect: Suppose that $\exists y \neq x$ such that $y \in(x+\mathbb{R} w) \cap X$. Then $y-x=\gamma w$ with $\gamma \neq 0$ and we obtain from the definition of $X$ that

$$
y \in \partial C+0, y \in \partial C+\alpha w, y \in \partial C+\gamma w, y \in \partial C+(\alpha+\gamma) w .
$$

Since $0 \neq \alpha \neq \gamma \neq 0$ three of the four numbers $0, \alpha, \gamma, \gamma+\alpha$ must be pairwise different which contradicts together with the fact that $y \in X$ Proposition 1 . So (3) has been proved. 
Let $Z:=\left\{z \in \mathbb{R}^{m} \mid \inf _{\{x \mid z-x \in \mathbb{R} w\}}\|x\|_{2}=1\right\}$ and let $S:=\left\{z \in Z \mid\|z\|_{2}=\right.$ $1\}$. We suppose without loss of generality that $0 \in \operatorname{int}(C \cap(C+\alpha w))$. Then $X \cap \mathbb{R} w=\emptyset$ and thus $X \backslash \mathbb{R} w=X$. We denote by $p r: X \rightarrow Z$ the projection of $X$ along the rays from the origin to $Z$. By the fact that $X \backslash \mathbb{R} w=X$ the mapping $p r$ is well defined. We identify $Z=S+\mathbb{R} w$ with $S \times \mathbb{R}$ and denote by $\mu:=o_{S} \otimes \lambda_{1}$ the measure on $Z$ which is the product of the surface area measure $o_{S}$ on $S$ and the Lebesgue measure $\lambda_{1}$ on $\mathbb{R}$. Note that by (3) we get for $x, \tilde{x} \in X$ that

$$
\begin{aligned}
& x \neq \tilde{x} \text { implies that for }(s, r),(\tilde{s}, \tilde{r}) \in S \times \mathbb{R} \text { with } \\
& (s, r)=\operatorname{pr}(x) \text { and }(\tilde{s}, \tilde{r})=\operatorname{pr}(\tilde{x}) \text { we have } s \neq \tilde{s} .
\end{aligned}
$$

Then $\mu(\operatorname{pr}(X))=\int_{S} \int_{\mathbb{R}} \mathbf{1}_{\operatorname{pr}(X)} d \lambda_{1} d o_{S}=0$ by Fubinis theorem and (4). Since $o_{C}$ is absolutely continuous with respect to $\mu \circ p r$ we obtain that $o_{C}(X)=0$ which proves the proposition.

We state now two further propositions without proofs.

Proposition 3 Given a convex body $C$ and a vector $w \in \mathbb{R}^{m} \backslash\{0\}$, then there exist two numbers $\alpha_{1}$ and $\alpha_{2} \in \mathbb{R}$ such that $C \cap\left(C+\alpha_{i}\right) \neq \emptyset$ and $\operatorname{int}(C \cap(C+$ $\left.\left.\alpha_{i}\right)\right)=\emptyset$. Further there exists a hyperplane $F$ such that $F \| C \cap\left(C+\alpha_{i}\right)$.

Proposition 4 Given a convex body $C$ and a vector $w \neq 0$ then

$$
o_{\partial C}\left\{x \in \partial C \text { s.t. }\left[H \| w \Rightarrow H \notin \nabla_{x} C\right]\right\}>0
$$

Lemma 1 Given a countable set $R \subset \mathbb{R}$, an element $\alpha_{0} \in R$, a convex body $C$, and a vector $w \in \mathbb{R}^{m} \backslash\{0\}$. Suppose that

$$
\left[\varsigma_{w}^{C} * \delta_{R}\right](B)=0 \text { for all Borel measurable sets } B \subseteq \partial\left(C+\alpha_{0} w\right) .
$$

Then the following conclusion holds:

There exists a hyperplane $F \nVdash w$ such that

$$
x \in \partial C \Rightarrow\left[\exists H \in \nabla_{x} C \text { s.t. } H \| w \text { or } \exists H \in \nabla_{x} C \text { s.t. } H \| F\right]
$$

Proof. We suppose without loss of generality that $\alpha_{0}=0$. Let

$$
Y=\left\{x \in \partial C \text { s.t. }\left[H \| w \Rightarrow H \notin \nabla_{x} C\right]\right\} .
$$

Then $o_{\partial C}(Y)>0$ by Proposition 4. Since there exists a version of $\frac{d \varsigma_{w}^{C}}{d o_{\partial C}}(y)$ such that $0 \neq \frac{d \varsigma_{w}^{C}}{d o_{\partial C}}(y)$ for all $y \in Y$ we get that

$$
\left.\varsigma_{w}^{C}\right|_{Y} \neq 0 .
$$

By Proposition 2 we obtain that if $C \cap(C+\alpha w)$ is a convex body and $0 \neq \alpha$ then

$$
\begin{gathered}
o_{\partial(C+\alpha w)}(Y)=o_{\partial(C+\alpha w)}(Y \cap(C+\alpha w))= \\
o_{\partial(C+\alpha w)}\left(\left\{x \mid x \in \partial C \cap(\partial C+\alpha w) \text { s.t. }\left[H \| w \Rightarrow H \notin \nabla_{x} C\right]\right\}\right)=0 .
\end{gathered}
$$


Since $\varsigma_{w}^{C} * \delta_{\alpha w}=\varsigma_{w}^{C+\alpha w}<<o_{\partial(C+\alpha w)}$ we obtain from (7) that $\left.\varsigma_{w}^{C} * \delta_{\alpha w}\right|_{Y}=0$ if $C \cap(C+\alpha w)$ is a convex body and $0 \neq \alpha$. Of course $\left.\varsigma_{w}^{C} * \delta_{\alpha w}\right|_{Y}=0$ holds also if $C \cap(C+\alpha w)=\emptyset$. Thus we obtain for $\alpha \neq 0$ that

$$
\left.\varsigma_{w}^{C} * \delta_{\alpha w}\right|_{Y}=0 \text { if } C \cap(C+\alpha w)=\emptyset \text { or } \operatorname{int}(C \cap(C+\alpha w)) \neq \emptyset .
$$

Since $R$ is countable we obtain from (5), (6) and since we supposed $\alpha_{0}=0$ that

$$
\left.\sum_{\alpha \in R \backslash\{0\}} \varsigma_{w}^{C+\alpha w}\right|_{Y}=\left.\sum_{\alpha \in R \backslash\{0\}} \varsigma_{w}^{C} * \delta_{\alpha w}\right|_{Y}=\left.\varsigma_{w}^{C} * \delta_{R \backslash\{0\}}\right|_{Y}=-\left.\varsigma_{w}^{C}\right|_{Y} \neq 0
$$

By Proposition 3 we obtain that there exist exactly two values $\alpha_{1}, \alpha_{2} \in \mathbb{R}$ such that $C \cap\left(C+\alpha_{i} w\right) \neq \emptyset$ and $\operatorname{int}\left(C \cap\left(C+\alpha_{i} w\right)\right)=\emptyset$ with $i \in\{1,2\}$. This implies together with (8) and (9) that

$$
\left.\sum_{i \in\{1,2\}} \varsigma_{w}^{C+\alpha_{i} w}\right|_{Y}=\left.\sum_{i \in\{1,2\}} \varsigma_{w}^{C} * \delta_{\alpha_{i} w}\right|_{Y}>>-\left.\varsigma_{w}^{C}\right|_{Y} .
$$

Since $\left.o_{\partial C}\right|_{Y}<<-\left.\varsigma_{w}^{C}\right|_{Y}$ we obtain from (10) that $o_{\partial C}$ almost all points of $Y$ must be contained in the union of $C \cap\left(C+\alpha_{1} w\right)$ and $C \cap\left(C+\alpha_{2} w\right)$. Further the sets $C \cap\left(C+\alpha_{1} w\right)$ and $C \cap\left(C+\alpha_{2} w\right)$ are by Proposition 3 parallel to one hyperplane $F$. The remaining null set $A \subset Y$ with respect to $o_{C}$ can not contain an open subset of $\partial C$. Thus $y \in A$ must be an element of $\overline{C \cap\left(C+\alpha_{1} w\right)}, \overline{C \cap\left(C+\alpha_{2} w\right)}$, or $\overline{\left\{x \in \partial C \mid \exists H \in \nabla_{x} C \text { s.t. } H \| w\right\}}$. In the first two cases there exists $H \in \nabla_{y} C$ s.t. $H \| F$ and in the third case there exists $H \in \nabla_{y} C$ s.t. $H \| w$. This concludes the proof.

Lemma 2 Given a convex body $C$ which fulfills the conclusion of Lemma 1. Then $C=C_{w}+[0, \beta] \cdot w$ for some real number $\beta>0$ and some convex set $C_{w} \nVdash w$ with $\operatorname{dim}\left(C_{w}\right)=m-1$.

Proof: Of course there exist exactly two hyperplanes $G^{1}$ and $G^{2}$ parallel to $F$ which support $C$.

Let $y \in \operatorname{int}(C)$. Then there exist reals $\alpha_{y}<\beta_{y}$ such that $y+\alpha_{y} w, y+\beta_{y} w \in$ $\partial C$. Since $y+\alpha_{y} w$ and $y+\beta_{y} w$ can not possess a hyperplane of support which is parallel $w$ they must by hypotheses possess a hyperplane of support parallel $F$. So we get, probably by an interchange of $G^{1}$ and $G^{2}$, that

$$
y+\alpha_{y} w \in G^{1} \cap C \text { and } y+\beta_{y} w \in G^{2} \cap C .
$$

By (11) it is clear that $\gamma=\beta_{y}-\alpha_{y}$ is independent of $y$ and $G^{2}=G^{1}+\gamma w$. Let $D^{1}=G^{1} \cap C$ and let $D^{2}=G^{2} \cap C$. Note that

$$
\begin{aligned}
& D^{1}=G^{1} \cap C=\overline{\left\{y+\alpha_{y} w \mid y \in \operatorname{int}(C)\right\}} \\
& D^{2}=G^{1} \cap C=\overline{\left\{y+\beta_{y} w \mid y \in \operatorname{int}(C)\right\}} .
\end{aligned}
$$


Thus $D^{1}$ and $D^{2}$ are compact convex sets of dimension $m-1$ with

$$
D^{2}=D^{1}+\gamma w .
$$

So we get by (11), (13), (12) and the compactness of $C$ that

$$
\operatorname{int}(C) \subset \operatorname{conv}\left(D^{1} \cup D^{2}\right)=D^{1}+[0, \beta] w \subseteq \bar{C}=C .
$$

Since $D^{1}+[0, \beta]$ is compact we get from (14) that $D^{1}+[0, \beta] w=C$. This completes the proof if we let $C_{w}=D^{1}$.

Lemma 3 Let $C=C_{i}+\left[0, \gamma_{i}\right] \cdot w_{i}$ for $\left(w_{1}, \ldots, w_{m}\right)$ linearly independent vectors in $\mathbb{R}^{m}$ and $C_{i} \not \mid w_{i}$ convex compact sets with $\operatorname{dim}\left(C_{i}\right)=m-1$. Then $C$ is a parallelepiped with 1-dimensional edges parallel with $w_{i}$.

Proof. Note that $\forall i \in\{1, \cdots, m\}$

$$
x \in \partial C \Rightarrow\left(x \in C_{i} \text { or } x \in C_{i}+\gamma_{i} \text { or }\left[H \in \nabla_{x}(C) \Rightarrow H \| w_{i}\right]\right) .
$$

From (15) we derive

$$
x \in \partial C \Rightarrow \exists i \text { s.t. }\left[x \in C_{i} \text { or } x \in C_{i}+\gamma_{i}\right] .
$$

Indirect: Otherwise by (15) $H \in \nabla_{x}(C)$ fulfills $H \| w_{i} \forall i \in\{1, \ldots, m\}$. Since $\left\{w_{i} \mid i=1, \ldots, m\right\}$ spans by hypotheses $\mathbb{R}^{m}$ no hyperplane can be parallel with all $w_{i}$. Thus we obtained a contradiction and (16) is proved.

Let $p r_{i}$ be the projection along $H$ onto $\mathbb{R} w_{i}$, i.e., let $p r_{i}(x)=y \in \mathbb{R} w_{i}$ s.t. $x-y \in H$. Then $C \subseteq \bigcap_{n=1}^{m} p_{i}^{-1}\left(p r_{i}(C)\right)=: D$ and $D$ is a parallelepiped which can for all $i=1, \ldots, m$ be written as $D=D_{i}+\left[0, \gamma_{i}\right] w_{i}$ with $D_{i} \supset C_{i}$.

So the lemma is proved if we show that $C=D$. We proceed indirect: Since $C$ is a convex body $C \neq D$ implies that there exists $x \in \partial C$ with $x \notin \partial D$. But by (16) there exists an $i \in\{1, \ldots m\}$ such that $x \in C_{i} \subseteq D_{i} \subset \partial D$ or $x \in C_{i}+\gamma_{i} w_{i} \subseteq D_{i}+\gamma_{i} w_{i} \subset \partial D$ which contradicts the fact that $x \notin \partial D$ and thus completes the proof of the lemma. $\square$

\section{Weak differentiation and a second Version of the Characterization Theorem}

Definition 3 We denote by $\mathcal{C}_{c}$ the space of continuous real valued functions with compact support on $\mathbb{R}^{m}$. We remark that a signed measure $\mu$ is determined by the integrals $\int \phi d \mu$ with $\phi \in \mathcal{C}_{c}$. Thus we can define the derivative of a set valued function as follows: Let $D:[0, \infty) \rightarrow \mathcal{P}\left(\mathbb{R}^{m}\right)$. If there exists a measure $\mu$ such that for any $\phi \in \mathcal{C}_{c}$

$$
\int \phi d \mu=\lim _{h \downarrow 0} \int \phi \frac{\mathbf{1}_{D(h)}-\mathbf{1}_{D(0)}}{h} d \lambda
$$


then we say that $\mu$ is the derivative from the right of $D($.$) at 0$. (We also say that $\mu$ is the weak limit $\lim _{h \downarrow 0} \frac{\mathbf{1}_{D(h)}-\mathbf{1}_{D(0)}}{h} \cdot \lambda$.)

Remark 2 The measure $\varsigma_{w}^{C}$ can also be described as follows: Let $H$ be the hyperplane perpendicular to $w$. Let $\lambda_{H}$ denote the $m$-1-dimensional Lebesgue measure on $H$. Let

$$
Y^{+}:=\{x \in \partial C \mid\langle\eta(x), w\rangle>0\}
$$

and

$$
Y^{-}:=\{x \in \partial C \mid\langle\eta(x), w\rangle<0\}
$$

Let $\mathrm{pr}_{+}: \mathrm{Y}^{+} \rightarrow H$ respectively $\mathrm{pr}_{-}: \mathrm{Y}^{-} \rightarrow H$ be the orthogonal projections onto $H$. Then we have for any Lebesgue measurable $B \in \mathbb{R}^{m}$ that

$$
\int_{x \in P}\|w\|_{2} \cdot \phi\left(p r_{+}^{-1}(x)\right) d \lambda_{H}(x)-\int_{x \in P}\|w\|_{2} \cdot \phi\left(p r_{-}^{-1}(x)\right) d \lambda_{H}(x)=\int \phi d \varsigma_{w}^{C}
$$

We note further that $p r_{+}\left(Y^{+}\right)=p r_{-}\left(Y^{-}\right)$and let $P:=p r_{+}\left(Y^{+}\right)=p r_{-}\left(Y^{-}\right)$. These facts can be established easily for Polytopes or convex bodies with differentiable boundary and then extend without difficulties to arbitrary convex bodies.

We calculate now the derivative from the right at 0 of the special set valued function $D(h)=C+h$, for a convex body $C$. (Compare with [8] Example 1.)

Proposition 5 Let $C$ be a convex body then the derivative from the right of $h \mapsto(C+h \cdot w)$ equals $\varsigma_{w}^{C}$, i.e.

$$
\int \phi d \varsigma_{w}^{C}=\lim _{h \downarrow 0} \int \phi \frac{\mathbf{1}_{(C+h \cdot w)}-\mathbf{1}_{C}}{h} d \lambda
$$

Proof: We use the notation of Remark 2. Further we denote by $\lambda_{1}$ the Lebesgue measure on $\mathbb{R}$. Then we have for Borel measurable sets $\tilde{H} \subset H$ and $R \subset \mathbb{R}$ that

$$
\lambda\left(\tilde{H}+\frac{\omega}{\|\omega\|} R\right)=\lambda_{1} \otimes \lambda_{H}(R \times \tilde{H})
$$

We obtain that

$$
\begin{gathered}
\lim _{h \downarrow 0} \int_{\mathbb{R}^{m}} \phi \frac{\mathbf{1}_{(C+h \cdot w)}-\mathbf{1}_{C}}{h} d \lambda= \\
\lim _{h \downarrow 0} \int_{\xi, x \in \mathbb{R} \times H} \phi\left(x+\xi \frac{w}{\|w\|_{2}}\right) \frac{\mathbf{1}_{(C+h \cdot w)}-\mathbf{1}_{C}}{h}\left(x+\xi \frac{w}{\|w\|_{2}}\right) d\left[\lambda_{1} \otimes \lambda_{H}\right](\xi, x)= \\
\lim _{h \downarrow 0} \int_{x \in H} \int_{\xi \in \mathbb{R}} \phi\left(x+\xi \frac{w}{\|w\|_{2}}\right) \frac{\mathbf{1}_{(C+h \cdot w)}-\mathbf{1}_{C}}{h}\left(x+\xi \frac{w}{\|w\|_{2}}\right) d \lambda_{1}(\xi) d \lambda_{H}(x)= \\
\int_{x \in P}\left[\lim _{h \downarrow 0} \frac{1}{h} \int_{\xi \in\left[0, h \cdot\|w\|_{2}\right]} \phi\left(p r_{+}^{-1}(x)+\xi \frac{w}{\|w\|_{2}}\right) d \lambda_{1}(\xi)\right] d \lambda_{H}(x)- \\
-\int_{x \in P}\left[\lim _{h \downarrow 0} \frac{1}{h} \int_{\xi \in\left[0, h \cdot\|w\|_{2}\right]} \phi\left(p r_{-}^{-1}(x)+\xi \frac{w}{\|w\|_{2}}\right) d \lambda_{1}(\xi)\right] d \lambda_{H}(x)=
\end{gathered}
$$




$$
\int_{x \in P}\|w\|_{2} \cdot \phi\left(p r_{+}^{-1}(x)\right) d \lambda_{H}(x)-\int_{x \in P}\|w\|_{2} \cdot \phi\left(p r_{-}^{-1}(x)\right) d \lambda_{H}(x)=\int \phi d \varsigma_{w}^{C}
$$

The first equality sign holds by (17), the second by Fubinis Theorem, the fourth by the main theorem of calculus and the last equality sign follows by Remark 2.

If we denote by $\mathbf{U}(C+h \cdot w)$ the uniform probability distribution on $C+h \cdot w$ and note that

$$
\int \phi \frac{\mathbf{1}_{(C+h \cdot w)}-\mathbf{1}_{C}}{h} d \lambda=\lambda(C) \cdot \int \phi d \frac{\mathbf{U}(C+h \cdot w)-\mathbf{U}(C)}{h}
$$

then we can reformulate Proposition 5 as follows:

Corollary 1 Let $C$ be a convex body. Then the weak limit $\lambda(C) \cdot \lim _{h \rightarrow 0} \frac{\mathbf{U}(C+h \cdot w)-\mathbf{U}_{(C)}}{h}$ equals $\varsigma_{w}^{C}$, i.e.,

$$
\lambda(C) \cdot \lim _{h \rightarrow 0} \int \phi d \frac{\mathbf{U}(C+h \cdot w)-\mathbf{U}(C)}{h}=\int \phi d \varsigma_{w}^{C} \text { for all } \phi \in \mathcal{C}_{c} .
$$

With Corollary 1 we can restate Theorem 1 as follows:

Theorem $2 A$ convex body $C \subset \mathbb{R}^{m}$ is a parallelepiped with one dimensional edges parallel $\left\{w_{1}, \ldots, w_{m}\right\}$ if and only if there exists a linearly independent set $\left\{w_{1}, \ldots, w_{m}\right\}$ of vectors $w_{j} \in \mathbb{R}^{m}$ such that for any $j \in\{1, \ldots, m\}$ there exists a countable set $R \subset \mathbb{R}$ and an $r_{0} \in R$ such that

$$
\left[\lambda(C) \cdot \lim _{h \rightarrow 0} \frac{\mathbf{U}(C+h \cdot w)-\mathbf{U}(C)}{h} * \delta_{\left\{r \cdot w_{j} \mid r \in R\right\}}\right](B)=0 .
$$

for any Borel measurable set $B \subseteq \partial C+r_{0} \cdot w_{j}$.

\section{References}

[1] Buchman, E.; Valentine, F.A. A characterization of the parallelepided in $E^{n}$ Pac. J. Math. 35, 53-57 (1970).

[2] Gruber, P.M.(ed.); Wills, J.M.(ed.) Handbook of convex geometry. Volume A. Amsterdam: North-Holland (1993).

[3] Guggenheimer, H.; Lutwak, E. A characterization of the n-dimensional parallelotope. Am. Math. Mon. 83, 475-478 (1976).

[4] Lehmann, E.L. Comparing location experiments. Ann. Stat. 16, No.2, 521-533 (1988).

[5] Pflug, Georg Optimisation of Stochastic Models. Kluwer Academic Publishers, Boston, 1996. 
[6] Phelps, Robert Convex Functions, Monotone Operators and Differentiability. 2nd Edition, Springer Verlag, Berlin Heidelberg (1993)

[7] Valentine, Frederick A. Convex sets (McGraw-Hill Series in Higher Mathematics) New York-San Francisco-Toronto-London: McGraw-Hill Book Company. (1964).

[8] Weisshaupt, Heinz A measure-valued approach to convex set-valued dynamics. Set-Valued Anal. 9, No.4, 337-373 (2001)

[9] Weisshaupt, Heinz A generalization of Lehmann's Theorem on the comparison of uniform location experiments

EURANDOM Report series Report 2003-014

http://www.eurandom.tue.nl/publications.htm 